CURIOUS COMPLICATION OF PARTURITION.

\author{
By W. H. BORHAM, L.R.C.P., M.R.C.S., \&c.
}

IN the " Clinical Notes" of THE LANCET of March 3rd, 1888, a case is narrated by Mr. Launcelot Archer of " Curious Contplication of Parturition." After detailing the case as he found it, and giving his treatment and describing the fotal malformation of the child after its birth, he asks a question. Before answering the question upon such an occult point, I venture to make a few preliminary remarks suggested by his case.

Mr. Archer states that the "os was fully dilated, the face presenting; the pains were frequent and strong, but quite ineffectual in bringing the head down." There is much ambiguity and misconception in the minds of young accoucheurs in their early career as to what constitutes a fully dilated os uteri in labour, and many are apt to think that an os is fully dilated when it is not so. My view of a fully dilated os is where its structure opens and becomes coeval and coequal to the capacity of the vaginal canal, sufficiently open to admit of the exit of the normal head of a child. A soft and dilatable os that the fingers are able to expand to the diameter of two inches and a half is not a fully dilated os, and will not always admit of a digital examination sufficient to explore, and lead one to recognise a facial presentation. Young practitioners are often exceedingly embarrassed in diagnosing the true presentation of a child (especially in such a condition of the os as above stated, where it can be expand only two inches and a half by the fingers), and where malformed heads, such as described in Mr. Archer's case, with swelling on the vertex, with the occipital bones divided, and large sulci between them; such heads, although presenting naturally, are often mistaken for tace presentation. Cases of breech previa are especially apt to be mistaken for such, and sometimes those of shoulder prævia have led one into the same error; and such cases do not reveal to the accoucheur the real state of things till the os has become fully dilated, and it is only then that certainty as to the presentation can be detected. I have known a young accoucheur stoutly adhere to his opinion all through a labour that in a certain case he felt sure it was a face presentation until he was confronted with the nates and its appendages issuing from the vagina. A careful manipulation with a welldirected and tutored hand, by a skilful and sensitive touch upon the structures of the presenting parts, are needful to indicate in many occult cases the true presentation of a child when the mother is in labour. Mr. Archer waited patiently for two hours, and as there was no progress made in the labour he resorted to the forceps, but could not get them to grip sufficiently tight upon the child's head to enable him to use enough traction to deliver. This he repeated several times unsuccessfully. The child had a hydrocephalic head; it was without parietal bones, with fluid on the brain, and the other cranial bones were in a shifting state. This complication in the child's head did not give it sufficient resistance to allow the forceps to hold on, and the blades slipped. It was like applying the forceps to a partly filled bladder of water-the more you squeezed the forceps together the greater was the bulging and bagging of the fluid contents of the cranium through the openings between the blades, rendering traction impossible. Thus foiled in delivering with the forceps, Mr. Archer very sensibly resorted to version, and delivered.

Mr. Archer asks this question: "How would one be able to diagnose a condition like this before birth, so as to avoid the attempt to deliver by forceps?" Applying the forceps several times in the same labour without any good result is very discouraging to the mother and embarrassing to the accoucheur, and often leaves (unfairly) an impression on the mind of the patient and attendants that a want of skill has been manifested on the part of the surgeon. I can sympathise with the query. If confronted in attend ance upon such a complicated case, my treatment would be very guarded; for I know that rare and unusual cases in midwifery are constantly cropping up, and we must watch, like the sea- and weather-wise sailor, and look out for "breakers ahead." I should press with the fingers upon the facial bones \&c., and see how much force was required before the structures were pushed up, and endeavour to ascertain how and in what direction they moved; then I should gradually force up between the pains the child's head into the uterus, then introduce the hand and carefully explore by gently manipulating the head with the fingers. By this means the malformation would be detected. This is the only possible means of diagnosing such a complicated case before delivery. Then I should do as Mr. Archer did-turn, which is a convincing process, showing the value of the hand above the forceps. We never know when new and unknown complications in nidwifery may arise, and it seems strange that this case should have offered such powers of resistance to the strong pains of the uterus. Such resist. ance could not have been owing to the face presentation, from the absence of the parietal bones, and the loose adaptation of the occipital bones, with the base of the brain containing clear fluid, and a thin membrane of dura mater covering it; in such a condition of the head its whole part nust have been very soft and yielding in every direction, and the facial bones would, in consequence of their loose attachments, have easily given way and moulded themselves to the soft parts of the mother by the strong uterine pains; therefore, it is clear this face presentation was not the factor of resistance. Another cause must be sought: Was it the deficiency in the cervical vertebræe? This might have caused the neck to be doubled up by the vis a tergo of the uterine pains, and thus produce obstruction. Or was the impediment to expulsion owing to an unusually large chest and shoulders impacted in the outlet of the pelvis? or from a very short funis? For myself, I should be inclined to attribute the obstacle in this case to the expulsion of the child by the normal efforts of the uterus to the deformity in the child's neck, whereby it became limpid, doubled up, or squeezed, blocking the way; and thus causing greater resist. ance than the power of the labour pains could overcome. Fulham.

A CASE OF

\section{TRAUMATIC EPILEPSY SUCCESSFULLY TREATED BY TREPHINING.}

BY F. A. SOUTHAM, M.B.OxON., F.R.C.S., ASSISTANT SURGEON TO THE MANCHESTER ROYAL INFIRMARY, AND SURGEON TO THE CLINICAL HOSPITAL FOR WOMEN AND CHILDREN.

WILLIAM $\mathrm{F}$ - , aged thirty-two, telegraphist. came under my care as an out-patient at the Manchester Royal Infirmary in August, 1887, with the following history. In December, 1886, having previously enjoyed good health, he fell from a height of sixteen feet, and sustained an injury to the head in the left temporal region, apparently a severe scalp wound without any fracture. He was removed in an unconscious condition to a local hospital, where he remained for about three weeks. At the end of this period he was discharged, having quite recovered from the effects of the accident. Shortly afterwards he began to suffer from attacks of a somewhat peculiar nature. Without any warn. ing, he would suddenly be seized with twitchings limited to the muscles of the right side of the face, and almost simultaneously he would become dizzy and fall to the ground in a state of complete unconsciousness, remaining in this condition for a varying period, often for several hours. In the intervals there was no evidence of paresis in the muscles of the face or of any other part of the body; speech was not affected, and there was no loss of sensation or impairment of any of the special senses. The attacks recurred at irregular intervals, sometimes as many as six daily, sometimes three or four a week; occasionally a week, but rarely longer, would pass without one. He again sought treatment at the same hospital, and underwent two operations, which from his description consisted in liberating the scar on the scalp by dissecting it up from the bone beneath; each operation was followed by a temporary cessation of the fits, which, however, soon recurred.

The man was suffering from a continuance of the same symptoms, which completely incapacitated him from following his occupation, when lie came under my observation. On examination, a well-marked adherent cicatrix about an inch in length was found in the left temporal region; no depression could be felt in the bone beneath, indicating that there had been a fracture. He was put on a course of 